\title{
Histologic features are important prognostic indicators in early stages lung adenocarcinomas
}

\author{
Joon Yim ${ }^{1}$, Lee-Ching Zhu ${ }^{1}$, Luis Chiriboga ${ }^{1}$, Heather N Watson ${ }^{2}$, Judith D Goldberg ${ }^{2}$ and \\ Andre L Moreira ${ }^{1}$ \\ ${ }^{1}$ Department of Pathology, New York University School of Medicine, New York, NY, USA and ${ }^{2}$ Department of \\ Environmental Medicine, Division of Biostatistics, New York University School of Medicine, New York, \\ NY, USA
}

\begin{abstract}
This study attempts to evaluate the clinicopathologic features of mixed subtype adenocarcinomas and the prognostic implications of histopathology classifications. Surgical specimens from 141 patients with clinical stage I or II lung adenocarcinoma during the period 1992-2004 were included. These cases were classified into four groups defined by the extent of the bronchioloalveolar carcinoma component: group I: pure bronchioloalveolar carcinoma; group II: mixed subtype with predominant bronchioloalveolar carcinoma component and $\leq \mathbf{5 m m}$ invasive component; group III: mixed subtype with bronchioloalveolar carcinoma component and $>5 \mathrm{~mm}$ invasive component; group IV: invasive carcinoma with no bronchioloalveolar carcinoma component. Descriptive statistics were used to examine the groups with respect to age, tumor size, lymph node metastasis, and Ki-67 and p53 expression levels. Death rate for the groups was obtained by patient's charts and from the National Death Index database. The population was similar in age, tumor size and lymph node metastasis. Immunohistochemical results showed that the mean Ki-67 labeling and the amount of p53 overexpression had the same trend of increasing mean values or positive results from groups I to IV. The reported proportion of deaths ranged from $0 \%$ for groups I and II, $20 \%$ in patients with predominant invasive component with bronchioloalveolar carcinoma (group III), and $18 \%$ in patients with invasive carcinomas and no bronchioloalveolar carcinoma component (group IV). The difference between the proportion of patients with reported deaths in the time period of this study in the combined greater than $5 \mathrm{~mm}+$ pure invasive groups (groups III, IV), and the $<\mathbf{5 m m}+$ noninvasive groups (groups I, II) is statistically significant. These results suggest that histological features may be useful in defining categories of lung adenocarcinomas with differing survival and prognostic features. These results are helpful in defining a subcategory of 'minimally invasive adenocarcinoma', which has features similar to bronchioloalveolar carcinoma.
\end{abstract}

Modern Pathology (2007) 20, 233-241. doi:10.1038/modpathol.3800734; published online 22 December 2006

Keywords: lung adenocarcinoma; bronchioloalveolar carcinoma; prognosis; histological classification

In the 2004, World Health Organization (WHO) classification of lung tumors ${ }^{1}$ a bronchioloalveolar carcinoma is a non-invasive carcinoma with a pure lepidic growth pattern. Solitary bronchioloalveolar carcinoma have a very good prognosis with reported 5 -years survival of $100 \% .{ }^{1,2}$ In the WHO classification, adenocarcinomas with different histological components, including bronchioloalveolar carcinoma were classified as adenocarcinoma with mixed subtypes. Controversy exists concerning the impact

Correspondence: Current address. Dr AL Moreira, MD, PhD, Department of Pathology, Memorial Sloan Kettering Cancer Center, 1275 York Avenue, New York, NY 10021, USA.

E-mail: moreiraa@mskcc.org

Received 28 June 2006; revised 13 November 2006; accepted 14 November 2006; published online 22 December 2006 of the histological classifications including the amount of bronchioloalveolar carcinoma in adenocarcinomas with mixed subtypes and prognosis.

Noguchi et $a l^{2}$ looked at small lung adenocarcinomas measuring $<2 \mathrm{~cm}$, and defined six different categories including pure bronchioloalveolar carcinoma, bronchioloalveolar carcinoma with areas of fibroblastic foci due to structural collapse, bronchioloalveolar carcinoma with active fibroblastic proliferation, and different categories of invasive carcinoma. They showed that patients with tumors with a pure bronchioloalveolar carcinoma pattern had a 100\% 5-year survival, patients with adenocarcinomas of mixed subtype with bronchioloalveolar carcinoma and invasive components had a $75 \%$ 5 -year survival, while those patients with a purely invasive carcinoma had a $52 \%$ 5-year survival. 
Yokose et $a l^{3}$ studied 200 cases of small lung adenocarcinomas ( $3 \mathrm{~cm}$ or less), and found that in tumors with more than $75 \%$ lepidic growth pattern, with central focus of fibrosis $\leqslant 5 \mathrm{~mm}$, and no elastic fiber framework destruction, no patient deaths were observed. Suzuki et $a l^{4}$ looked at the size of scars seen in peripheral adenocarcinomas, and showed that there was $100 \% 5$-year survival if the scar was $5 \mathrm{~mm}$ or smaller, $72 \%$ if the scar was between 5 and $15 \mathrm{~mm}$, and $40 \%$ if the scar was $>15 \mathrm{~mm}$.

However, a study by Ebright et al, ${ }^{5}$ indicated that dividing adenocarcinomas into different groups, including bronchioloalveolar carcinoma, bronchioloalveolar carcinoma with focal invasion, adenocarcinoma with bronchioloalveolar carcinoma features, and pure invasive carcinoma, showed no difference in 5-year survival, but it was the clinical pattern (defined as unifocal, multifocal disease, or pneumonic pattern radiographically) and pathologic staging that were important indicators for prognosis.

It is worth noting that all studies that showed an impact of the histological classification on prognosis, came from the same geographic region, which could suggest ethnic difference in biological behavior or differences in the management of lung lesions. ${ }^{2-4,6,7}$ It was imperative, therefore to evaluate the same criteria in a population genetically heterogeneous such as the one in the US.

In this study, we evaluated small adenocarcinomas in an attempt to validate previous reports that the amount of invasive component in adenocarcinomas, mixed subtypes, has prognostic significance. All cases studied were restricted to patients with clinical stages I and II.

We classified small pulmonary adenocarcinomas into four histologically defined categories: group I: composed of pure bronchioloalveolar carcinoma, group II: composed of adenocarcinoma, mixed subtypes with bronchioloalveolar carcinoma and invasive component, where the area of invasion measured $\leqslant 5 \mathrm{~mm}$ in diameter, group III: adenocarcinoma, mixed subtype with bronchioloalveolar carcinoma and invasive component, where the area of invasion measured more than $5 \mathrm{~mm}$ in diameter, and group IV: adenocarcinomas, mixed subtypes composed of purely invasive types with no bronchioloalveolar carcinoma component. We then compared different clinicopathologic features of the four groups, including patient age, tumor size, presence of lymph node metastasis, mortality rates, proliferative potential (Ki-67), and frequency of P53 abnormalities, in order to assess if these histologic markers had any significance in prognosis.

\section{Materials and methods}

\section{Patient Population}

In this study, we reviewed surgical specimens from all of the 141 patients diagnosed with lung adeno- carcinoma, surgically resected with curative intent at New York University Medical Center during the period of 1992-2004. All cases were from patients with clinical American Joint committee on Cancer (AJCC) stage I or $\mathrm{II}^{8}$ None of the patients included in this study had received preoperative chemotherapy or radiotherapy. The female to male ratio was 1.3:1.

Patient data were collected according to Health Insurance Portability and Accountability Act (HIPAA) regulations. The study was approved by the New York University Institutional Review Board.

\section{Histological Review}

The surgically resected tumors were fixed in $10 \%$ formalin for $2-5 \mathrm{~h}$. The tumors were cut at $2 \mathrm{~mm}$ intervals, and representative sections or entire tumor were submitted for microscopic evaluation. The paraffin-embedded tissues were sliced into $3-\mu \mathrm{m}$ sections and stained with hematoxylin and eosin. All slides were reviewed by two pathologists (JY, ALM) and were classified using the criteria set forth by the 2004 World Health Organization classification of lung tumors. ${ }^{1}$ Discrepancies were resolved by consensus in a joint review of the slides at a multihead microscope. Most of the discrepancies occurred among the bronchioloalveolar carcinoma group with respect to the presence or absence of an invasive component. Pulmonary adenocarcinomas were divided into four different groups. Group I consisted of adenocarcinomas with a pure bronchioloalveolar pattern showing no evidence of stromal, vascular, or lymphatic invasion. None of the bronchioloalveolar carcinoma studied were of the mucinous type.

Group II consisted of adenocarcinomas, mixed subtypes, with bronchioloalveolar carcinoma and invasive carcinoma, where the invasive component measured less than or equal to $5 \mathrm{~mm}$ in diameter. If the focus of invasive carcinoma was seen in the middle of an area of fibrosis, the entire area of fibrosis was considered in the measurement of the diameter. If the invasive carcinoma was seen at the periphery of an area of central fibrosis with bronchioloalveolar carcinoma component seen surrounding this area, then the measurement was taken from the interface of the bronchioloalveolar carcinoma area and the central fibrosis including the invasive carcinoma (Figure 1).

Group III consisted of adenocarcinomas, mixed subtypes, with bronchioloalveolar carcinoma and invasive carcinoma, where the invasive component measured more than $5 \mathrm{~mm}$ in diameter.

Group IV consisted of adenocarcinomas mixed subtypes, with purely invasive patterns and no bronchioloalveolar carcinoma component.

The invasive components present in the mixed subtype adenocarcinomas were acinar, papillary, 


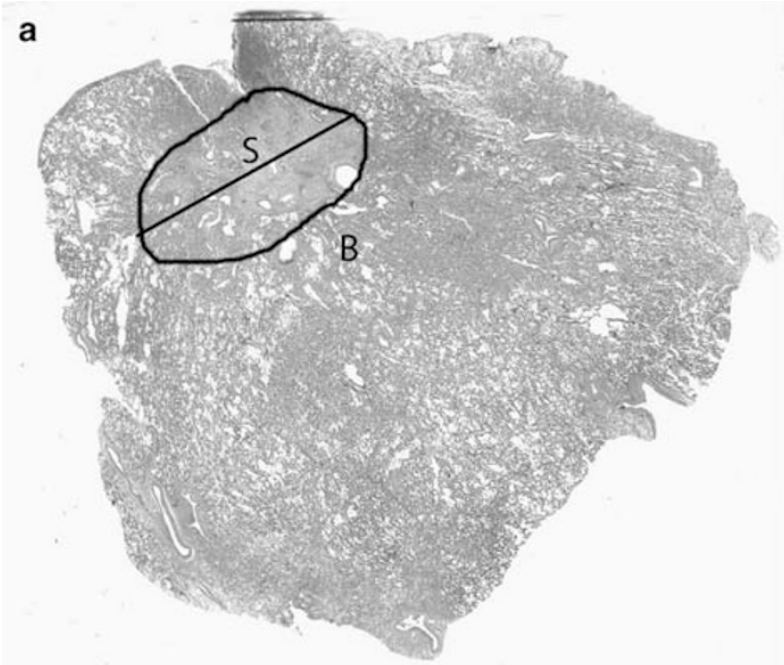

b

Figure 1 Examples of how the measurement of the area of invasion was obtained. (a) In this case of a predominant BAC component (B) the area of invasion is circled, it consists mostly of scar tissue (S). The measurement followed the greatest dimension of invasion (strait line). In (b), the area of invasion is composed of scar tissue (S) and invasive adenocarcinoma (I) that does not induce scarring. Similarly to (a), the measurement followed the greatest dimension marked by a strait line.

micropapillary, and solid, or more commonly, a combination of different histological patterns.

\section{Clinical Data}

The patient age and tumor size were obtained from the corresponding surgical pathology report for each specimen. The presence or absence of lymph node metastasis was also determined from the surgical pathology report along with confirmatory slide review of all cases.

\section{Immunohistochemical Stains}

Immunohistochemistry for $\mathrm{P} 53$ and Ki-67 were performed in 41 and 39 cases, respectively. Cases were selected for immunohistological study according to the amount of tissue available in the block. Care was taken not to exhaust the blocks. Paraffinembedded tissue was cut in 5 - $\mu$ m-thick sections, and from appropriate control tissues. The sections were deparaffinized and submitted to antigen retrieval by heating in citrate buffer, $\mathrm{pH}$ 6.0, 5$10 \mathrm{~min}$, the sections were then incubated with primary antibodies Ki-67 (1:20 dilution; clone MIB-1; DakoCytomation) and P53 (1:50 dilution; clone DO-1; Santa Cruz Biotechnology). The immune complexes were detected by a modified avidin-biotin-peroxidase technique in a semiautomated staining instrument (Ventana Medical System, Tucson, AZ, USA).

The Ki-67 expression was determined by counting 500 tumor cells in three high-power fields $(\times 400)$ from the most highly labeled areas and the results are expressed as percentage of positive cells. Positive p53 overexpression was defined as the presence of nuclear staining in $>20 \%$ of tumor cells. ${ }^{9}$

\section{Patient Survival}

Survival of patients from all groups was assessed from review of medical records and from the National Death Index database reported as of 31 July 2004.

\section{Statistical Analysis}

The main objective of this study is to examine the association between disease group and survival. Patients for whom no date of death was obtained from the death records are censored. As there may be a delay in reporting of deaths, recent deaths may not be included. Descriptive statistics, including the proportion of cases in each class with each of the prognostic factors are provided. The proportion of reported deaths for each class is also provided. Fisher's Exact test was used for associations between group and reported death, lymph node metastasis, and p53 expression. To test the association between Ki-67 and group, the nonparametric WilcoxonMann-Whitney test is used because Ki-67 is not normally distributed among groups. Multiple logistic regression analysis is used to examine the associations of tumor size, lymph node metastasis, Ki-67 or group and the reported death.

Kaplan-Meier survival curves, that take into account the length of follow-up and time of death, are constructed based on a range of assumptions regarding the last reported observation date for censored patients. These assumptions include: last known date alive is the last or most recent recorded death date (31 July 2004) in the data set; midpoint between the last reported death date and the patient's date of diagnosis; censoring at date of diagnosis. Kaplan-Meier curves were compared among the four groups. 


\section{Results}

\section{Clinical Data}

A total of eight patients were classified into group I. This group included bronchioloalveolar carcinoma with no clear evidence of invasive carcinoma (Figure 2a). Twenty-one patients were classified into group II, an example of these tumors is illustrated in Figure 2b. Forty-six patients were placed into the group III category (Figure 2c). Acinar and papillary types adenocarcinomas were the most common invasive components of the tumors classified as groups II and III. Sixty-six patients were classified into group IV (Figure 2d). Within this group, acinar, papillary, solid and micropapillary types of invasive adenocarcinoma were present. Most tumors showed a mixture of histological components with acinar being the most frequent $(80 \%)$, and micropapillary, the least frequent $(12 \%)$.
The mean age of the patients was similar in each of the groups studied (Table 1). There was considerable overlap in the average size of the resected tumors in all four groups, although tumor size increased slightly from groups I to IV (Table 1).

All patients were staged at the time of surgery by regional lymph node dissection. Assessment of lymph node metastasis indicated no lymph node involvement in any of the patients from groups I and II at the time of surgery. In contrast, 6\% (three of 46) of group III patients showed lymph node metastasis (stage II), while $12 \%$ (eight of 66) of group IV patients had lymph node involvement (stage II) (Table 1). All positive lymph nodes were N1 nodes (ipsilateral peribronchial and/or hilar). The four disease groups were similar with respect to the proportion of patients with lymph node metastasis (Fishers Exact Test, $P=0.34,2$-sided). If the four groups are combined into two groups (groups I-II
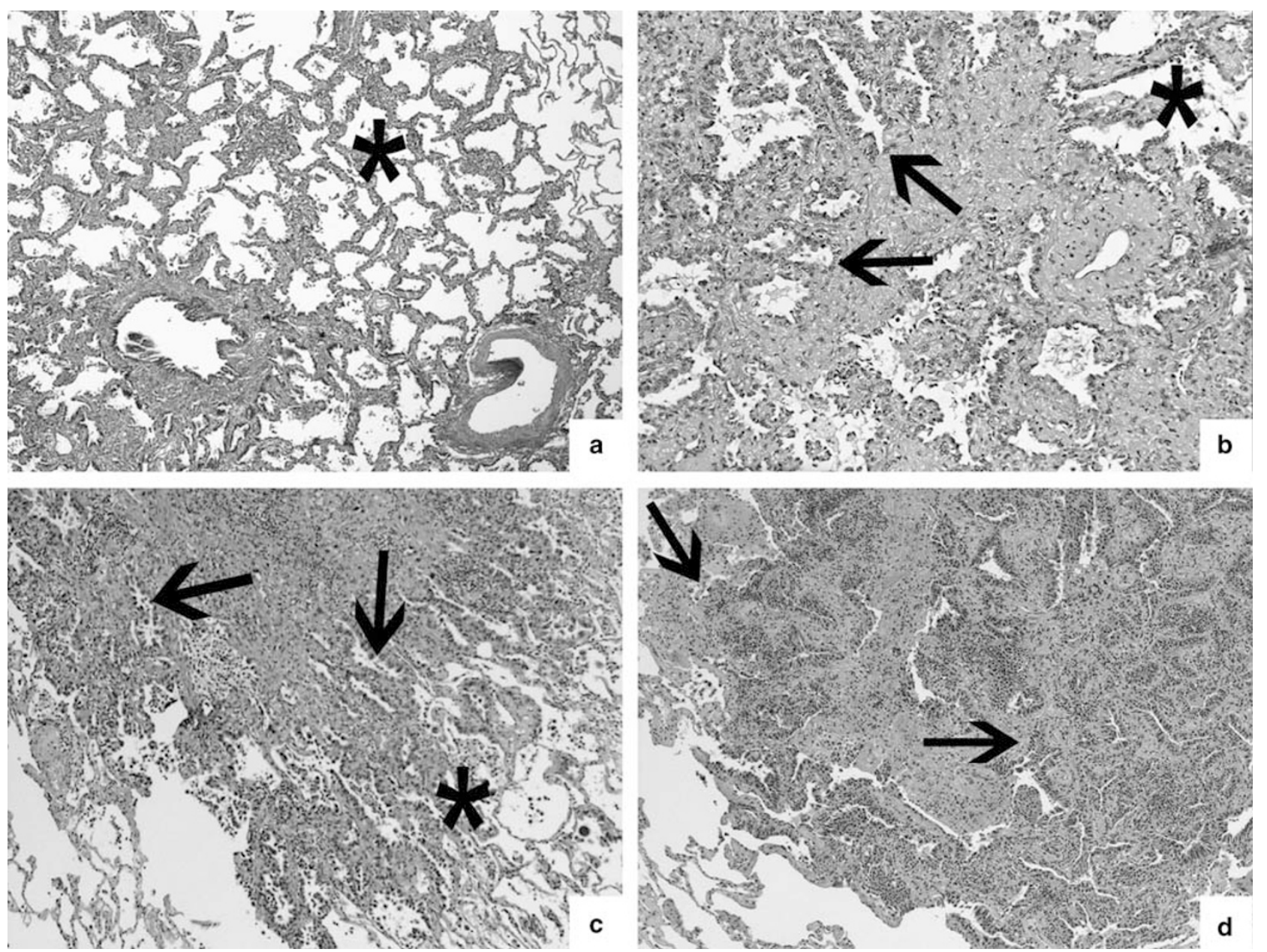

Figure 2 Microphotographs of examples of pulmonary adenocarcinomas within each groups studied (a) group 1, pure BAC, note the distended alveolar septa, lepidic growth (asterisk) and no scar, (b) group II, adenocarcinoma, mixed subtype with BAC and $<5$ mm area of invasive adenocarcinoma component; note small area of scar with acinar pattern (arrows), area of lipidic growth is marked with an asterisk. (c) Group III, adenocarcinoma, mixed subtype with BAC and more than $5 \mathrm{~mm}$ area of invasive component. Note larger area of scar. Arrows mark acinar pattern, an asterisk marks peripheral BAC pattern. (d) Group IV, adenocarcinoma mixed subtype with no BAC component, arrows mark invasive pattern of adenocarcinoma. 
Table 1 Patient demographic characteristics by histologic group

\begin{tabular}{lcccc}
\hline Group & Number of patients (\% total) & Age (mean \pm s.d.) & Tumor size (cm) & Lymph node metastasis (\% group) $^{\#}$ \\
\hline I & $8(6)$ & $67 \pm 3.9$ & $1.1 \pm 0.45$ & $0(0.0)$ \\
II & $21(15)$ & $64 \pm 9.6$ & $1.85 \pm 1.61$ & $0(0.0)$ \\
III & $46(33)$ & $67 \pm 9.7$ & $2.18 \pm 0.95$ & $3(6)$ \\
IV & $66(46)$ & $66 \pm 12.0$ & $2.77 \pm 2.23$ & $8(12)$ \\
\hline
\end{tabular}

\#The frequency of lymph node metastasis was not significantly different from groups I and II to groups III and IV $(P>0.05)$.

and III-IV), there is still no association between lymph node metastasis and group (Fishers Exact Test, $P=0.12,2$-sided).

\section{Immunohistochemical Results}

Immunohistochemistry was performed to evaluate the expression of Ki-67 and P53, since these markers have been reported to be independent prognostic markers in pulmonary adenocarcinomas. ${ }^{9-12}$ Among the 141 cases studied, 39 were evaluated for the expression of Ki-67 (four from group I, 11 from group II, 10 from group III, and 14 from group IV). Forty-one patients were evaluated for p53 staining. A representative section of the tumor was stained for the expression of Ki-67 and p53 (Figure 3). The mean Ki-67 expression levels increased from groups I to IV (Table 2). Positive expression of p53 increased with subsequent higher groups (Table 2).

A subset of 39 patients, who had both Ki-67 and p53 values was also evaluated. When we compared the subset of patients with both values in each of the four groups, those patients with both values recorded were older (on average) with smaller tumor size (on average). There was no difference in the proportion of patients with lymph node metastasis between the subset and the total population. In the subset population, Ki-67 levels differ for the four groups (Kruskal-Wallis $P=0.004$ ) and that difference of Ki-67 levels remains when the groups are divided into two (groups I and II; and groups III and IV). There is no association between p53 staining and the four disease groups (Fishers Exact, $P=0.34$, 2 -sided). Reducing to two groups does not change the lack of association between p53 staining and group.

\section{Survival Analysis}

An examination of the crude death rates by disease group suggest minimal differences among the four groups (Fishers Exact, $P=0.07,2$-sided). In particular, there are no deaths in groups I and II. When these two groups are compared with groups III and IV, there is a significant difference in the crude death rates among the three groups (Fishers Exact, $P=0.02$, 2-sided). However, there is no difference in the distribution of survival times between groups III and IV (Log-Rank, $P=0.37$ ). If the combined groups
(I, II) and (III, IV) are considered, then the crude death rates differ by disease group (Fishers Exact, $P=0.007,2$-sided). There was no association between lymph node metastasis and reported death (Fishers Exact, $P=1.0,2$-sided). None of the variables, including age, are significant in a multiple logistic regression model where the outcome is death or censored.

Follow-up for survival in these patients ranged from 23 days to 12.4 years, using 31 July 2004 as the most recent recorded death date. There were no operative deaths, that is, death within 30 days of surgical procedure.

Stratification of the patients into the four groups showed that there were no deaths due to the disease in patients from groups I and II (100\% survival). All patients in these groups were stage I. In patients with predominant invasive component with BAC (group III) $20 \%$ of the patients died of the disease (nine in 46 patients). In all, $18 \%$ of the patients in group IV (12 in 66) died of the disease, a group composed entirely of invasive carcinomas and no BAC component. Interestingly, $63 \%$ of the patients in group IV that had micropapillary pattern in their tumors died of the disease, in comparison to $27 \%$ of patients with solid pattern, $14 \%$ with acinar, and $10 \%$ with papillary patterns. Although the number of death in this study population was small for statistical analysis of a specific histological pattern and prognosis, our data seem to support previous observations ${ }^{13,14}$ that a micropapillary pattern seems to carry a worse prognosis than the other patterns of invasive adenocarcinoma.

Date of death was obtained for 21 patients from the National Death Index Database. Figure 4 provides the Kaplan-Meier survival curves for the four disease groups under the assumption that the last or most recent recorded death date (31 July 2004) is the last observation for censored patients.

As $92 \%$ of patients tested negative for lymph node metastasis, an additional analysis in the lymph node metastasis negative patients was conducted. The resulting Kaplan-Meier curves appear similar to the curves for all patients. Alternative assumptions about the last observation date for censored patients did not change the results.

The survival curves do appear different for groups ((III, IV) and (I, II)) using $5 \mathrm{~mm}$ as the cutoff. Group (I, II) has no deaths, which suggests that all of these patients were alive or their deaths had not been 

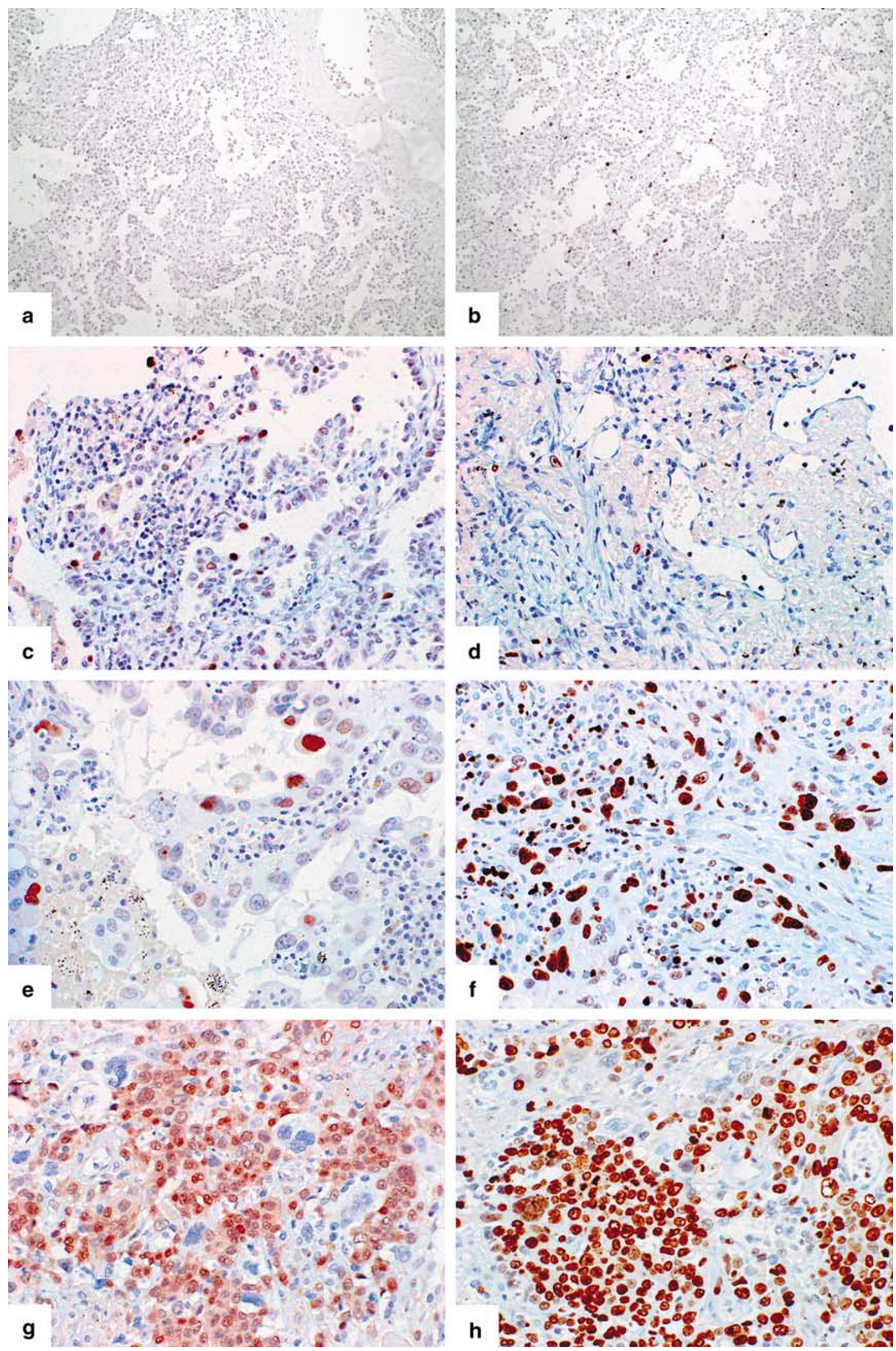

Figure 3 Examples of P53 and Ki-67 labeling of the tumor cells, respectively, for group I (a and b); group II (c and d); group III (e and f); and group IV (g and $\mathbf{h}$ ). The pictures for p53 and Ki-67 were taken from areas of most intense labeling. 
Table 2 Immunohistochemical characteristics by histologic group for a subset of patients

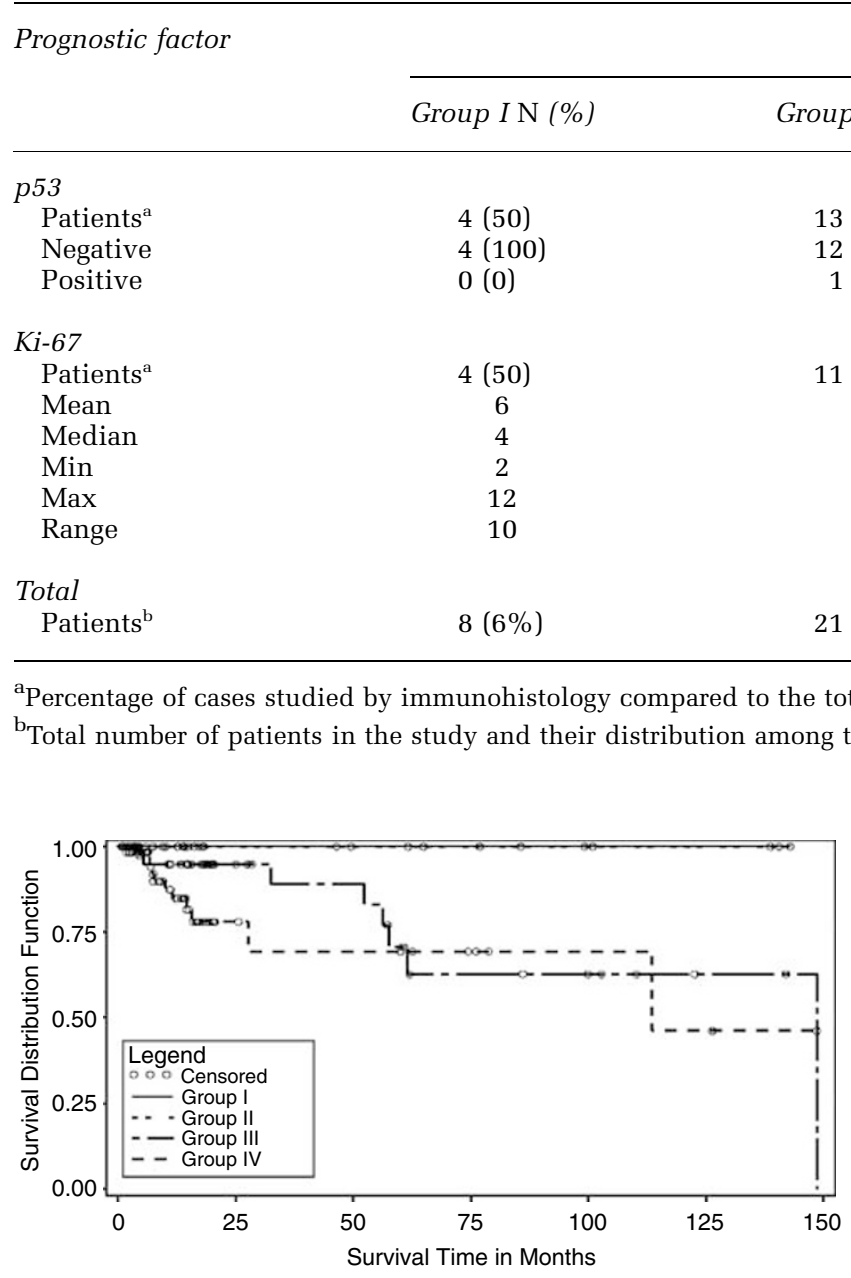

Figure 4 Kaplan-Meier survival curves by disease groups for patients.

entered in the database when investigators searched the National Death Index. In groups (III, IV) the median survival time (estimated from the KaplanMeier curve) is 10 years.

In addition, it was noted that in the first year of follow-up for patients in group III, the survival rate is $94 \%$; in contrast patients in group IV have $84 \%$ survival in the first year of follow-up.

\section{Discussion}

Recent developments in radiology have allowed for the detection of small peripheral lung cancers. Over the past year, there has been a number of publications on small pulmonary adenocarcinomas that have challenged the concept that these adenocarcinomas invariably carry a poor prognosis. This concept was initiated by Noguchi et $a l^{2}$ that showed that small peripheral adenocarcinomas of the lung with a pure pattern of bronchioloalveolar carcinoma had an excellent prognosis with $100 \%$ survival in
Pulmonary adenocarcinoma

Group III N (\%)

Group IV N (\%)

$13(61)$
$12(92)$
$1(8)$


$11(52)$
7
6
5
13
9

$1(15 \%)$

$10(21)$
$8(80)$
$2(20)$

$10(21)$
9
6
2
36
34

$14(21)$

$9(63)$

5 (37)

$14(21)$

23

17

64
58

$66(46 \%)$
5 years. A direct consequence of these observations was a change in the classification of pulmonary carcinomas by the World Health Organization that proposed bronchioloalveolar carcinoma as a separate category of adenocarcinoma. Bronchioloalveolar carcinoma is now defined as carcinoma with pure lepidic growth with no areas of invasion.

Several studies originating from Japan have shown that small adenocarcinomas mixed subtypes with a predominant bronchioloalveolar carcinoma component also have very good prognosis, comparable to pure bronchioloalveolar carcinoma. ${ }^{3,4,6,7,12}$ In these studies, the investigators have looked into different histopathological features as ways of identifying subgroups with different prognosis. Some have looked at the size and nature of the fibrosis, ${ }^{4,6,12}$ others have looked into the degree of lepidic growth. ${ }^{3}$

Criticism of these studies have been centered on the difficulty in reproducing the histological parameters used, as well as, the fact that the majority of these studied come from the same geographic region where bronchioloalveolar carcinoma is more frequent than in other areas of the globe. ${ }^{15}$ This increase in incidence of bronchioloalveolar carcinoma and adenocarcinomas with mixed subtypes with large amounts of bronchioloalveolar carcinoma may be a consequence of genetic differences in the population or differences in the clinical management of small pulmonary nodules. There are few reports from the US and Europe on the prognosis of bronchioloalveolar carcinoma. ${ }^{16-18}$ In these papers, bronchioloalveolar carcinoma has a better prognosis than invasive adenocarcinoma, with reduced recurrence rates and death, but not $100 \%$ survival. The differences in results may be due to differences in the classification of bronchioloalveolar carcinoma, since the studies were performed before the 2004 
WHO classification, in addition, there is no review of the histological material or comparison by stage of disease. ${ }^{16,17}$ In a recent consensus workshop for the classification of pulmonary carcinoma, a call for validation of the Japanese findings have been made. $^{15}$

The cases that were included in our study were obtained in the period of 12 years (1992-2004), and the percentage of pure bronchioloalveolar carcinoma in our series was $6 \%$. This is in agreement with published reports on the incidence of bronchioloalveolar carcinoma in the US and Europe. ${ }^{16,17}$ Our approach consisted of a simple and reproducible histological classification of small pulmonary adenocarcinomas based on the extent of the invasive component. Mixed subtype adenocarcinomas with bronchioloalveolar carcinoma features were separated into two groups, those with foci of invasion $<5 \mathrm{~mm}$ in diameter, and those with foci of invasion $>5 \mathrm{~mm}$ in diameter. Invasion was identified by any of the patterns of invasive adenocarcinoma, namely acinar, papillary, micropapillary, or solid, and the presence of fibrosis. In our approach, there was no distinction of the areas of fibrosis (central or peripheral) or quality of the fibrotic process. In all cases studied, the area of scar was always associated with an invasive pattern of adenocarcinoma, most commonly acinar pattern. There were no cases of bronchioloalveolar carcinoma with scar without an areas of invasion. By considering that any fibrosis is comparable to stromal invasion, we were able to identify two groups among the adenocarcinomas, mixed subtypes with bronchioloalveolar carcinoma component. These two subgroups were compared to pure bronchioloalveolar carcinoma and adenocarcinomas with pure invasive components. We also restricted our examination to patients with AJCC stages I and II tumors since it has been described that the good prognostic values of a pure bronchioloalveolar carcinoma is irrelevant when the disease presents as multifocal or in the pneumonic form. ${ }^{5}$

In defining different groups using the $5 \mathrm{~mm}$ cutoff, we found subgroups of mixed subtype lung adenocarcinoma with bronchioloalveolar carcinoma components with distinctive clinicopathological characteristics. By examining mortality rates, we observed no deaths in group II patients during the follow-up period, which is similar to patients in group I, as well as the reported survival data for pure bronchioloalveolar carcinoma in the Japanese studies. ${ }^{2-4,6,7}$ This data shows that within the category of adenocarcinomas with mixed subtypes with bronchioloalveolar carcinoma features, using the cutoff value of $5 \mathrm{~mm}$, we can define two distinct subcategories with different clinicopathologic characteristics and prognoses.

Similar findings were observed with the frequency of Ki-67 proliferation index, an independent prognostic markers in pulmonary adenocarcinomas. ${ }^{9,12} \mathrm{Ki}-67$ detects a nuclear membrane antigen, ${ }^{19}$ and its expression correlates with mitotic activity in many tumors. ${ }^{19,20}$ The Ki-67 labeling index of $30 \%$ has been associated with poor prognosis in pulmonary adenocarcinomas. ${ }^{12,21,22}$ In the present study, the Ki-67 labeling index increased from groups I to IV, although there was considerable overlap in the proliferative index, there was a trend of increased labeling with the severity of the carcinoma. The mean labeling index above $30 \%$ was seen only in tumor classified as group IV.

The product of the p53 gene is implicated in the control of different cell functions such a cell cycle, DNA repair, and cell differentiation. ${ }^{23}$ Abnormalities in p53 expression has been documented in pulmonary adenocarcinomas, ${ }^{24-26}$ and it has been suggested that increased labeling of tumor cells with p53 is associated with a poor prognosis. ${ }^{9,12,25}$ Of the tumors analyzed for p53 staining, those in groups III and IV had higher positive labeling for the marker than the other two groups, although in our study, the difference is not statistical significant.

Defining such distinct groups becomes important for management implications when we consider that more adenocarcinomas of the lung, which are being resected are in the category of mixed subtype adenocarcinomas with BAC features.

Our data is confirmatory in that patients with a pure bronchioloalveolar carcinoma, as defined by the new WHO classification of lung tumors, ${ }^{1}$ have different prognosis from patients with a purely invasive adenocarcinoma.

In fact, the data suggests that a subgroup (group II) of 'minimally invasive adenocarcinoma' could be defined. Although group II tumors had an invasive component, all the clinical and histopathological parameters evaluated (lymph node metastasis, p53 abnormality, Ki-67 frequency, mortality rate) were very similar to those in group I (pure bronchioloalveolar carcinoma).

Considering that approximately $20-50 \%$ of stage I lung cancer patients presents with recurrence of the disease within 5 years of surgery, ${ }^{27,28}$ and that approximately $30 \%$ of surgically treated patients with stage I lung cancer die of the disease within 5 years of surgery, ${ }^{28-30}$ the awareness of a minimally invasive adenocarcinoma of the lung with good prognosis may be an important factor in the clinical management of these patients.

\section{References}

1 Travis WD, Brambilla E, Muller-Hermelink HK, et al. Pathology and Genetics: Tumours of the Lung, Pleura, Thymus and Heart. World Health Organization Classification of Tumours. IARC press: Lyon, 2004.

2 Noguchi M, Morikawa A, Kawasaki M, et al. Small adenocarcinomas of the lung. Histologic characteristics and prognosis. Cancer 1995;15:2844-2852.

3 Yokose T, Suzuki K, Nagai K, et al. Favorable and unfavorable morphological prognosis factors in peripheral adenocarcinoma of the lung $3 \mathrm{~cm}$ or less in diameter. Lung Cancer 2000;29:179-188. 
4 Susuki K, Yokose T, Yoshida J, et al. Prognostic significance of the size of central fibrosis in peripheral adenocarcinoma of the lung. Ann Thorac Surg 2000; 69:893-897.

5 Ebright MI, Zakowski MF, Martin J, et al. Clinical pattern and pathological stage but not histologic features predict outcome for bronchioloalveolar carcinoma. Ann Thorac Surg 2002;74:1640-1647.

6 Sakurai H, Dobashi Y, Mizutani E, et al. Bronchioloalveolar carcinoma of the lung 3 centimeters or less in diameter: a prognostic assessment. Ann Thorac Surg 2004;78:1728-1733.

7 Sakurai H, Maeshima A, Watanabe S, et al. Grade of stromal invasion in small adenocarcinoma of the lung. Histological minimal invasion and prognosis. Am J Surg Pathol 2004;28:198-206.

8 Fleming ID, Cooper JS, Henson DE, et al. AJCC Cancer Staging Manual, 5th edn. American Cancer Society, Lippincot Williams \& Wilkins: Philadelphia, PA.

9 Ishida $\mathrm{H}$, Irie $\mathrm{K}$, Itoh $\mathrm{T}$, et al. The prognostic significance of p53 and bcl-2 expression in lung adenocarcinoma and its correlation with Ki-67 growth fraction. Cancer 1997;80:1034-1045.

10 Quinlan DC, Davidson AG, Summers CL, et al. Accumulation of P53 protein correlates with poor prognosis in human lung cancer. Cancer Res 1992; 52:4828-4831.

11 Fujino M, Dosaka-Akita H, Harada M, et al. Prognostic significance of p53 and ras p21 expression in nonsmall cell lung cancer. Cancer 1995;76:2457-2463.

12 Terasaki H, Niki T, Matsuno Y, et al. Lung adenocarcinoma with mixed bronchioloalveolar and invasive components. Clinicopathological features, subclassification by extent of invasive foci, and immunohistochemical characterization. Am J Surg Pathol 2003; 27:937-951.

13 Roh MS, Lee JI, Choi PJ, et al. Relationship between micropapillary components in the regional lymph nodes of patients with stage I lung adenocarcinomas. Histopathology 2004;45:580-586.

14 Makimoto Y, Nabeshima K, Iwasaki H, et al. Micropapillary pattern: a distinctive pathological marker to subclassify tumors with a significantly poor prognosis within small peripheral lung adenocarcinoma $(</=20 \mathrm{~mm})$ with mixed bronchioloalveolar and invasive subtypes (Noguchi's type C tumors). Histopathology 2005;46:677-684.

15 Travis WD, Garg K, Franklin WA, et al. Evolving concepts in the pathology and computed tomography imaging of lung adenocarcinoma and bronchioloalveolar carcinoma. J Clin Oncol 2005;23:3279-3287.
16 Zell JA, Ignatious Ou SH, Ziogas A, et al. Epidemiology of bronchioloalveolar carcinoma: improvement in survival after release of the 1999 WHO Classification of lung tumors. J Clin Oncol 2005;23:8396-8405.

17 Rena O, Papalia E, Ruffini E, et al. Stage I pure bronchioloalveolar carcinoma: recurrences, survival and comparison with adenocarcinoma of the lung. Eur J Cardiothoracic Surg 2003;23:409-414.

18 Okubo K, Mark EJ, Flieder D, et al. Bronchioloalveolar carcinoma: clinical, radiologic, and pathologic factors and survival. J Thorac Cardiovasc Surg 1999;118: 702-709.

19 Gerdes J, Lemke H, Baisch H, et al. Cell cycle analysis of a cell proliferation-associated human nuclear antigen defined by the monoclonal antibody Ki-67. J Immunol 1984;133:1710-1715.

20 Schwarting R. Little missed markers and Ki-67. Lab Invest 1993;68:597-599.

21 Pence JC, Kerns BJ, Dodge RK, et al. Prognostic significance of the proliferation index in surgically resected non-small cell lung cancer. Arch Surg 1993;128:1382-1390.

22 Scagliotti GV, Micela M, Gubetta L, et al. Prognostic significance of $\mathrm{Ki}-67$ labeling in resected non-small cell lung cancer. Eur J Cancer 1993;3:363-365.

23 Harris CC, Hollstein M. Clinical implications of the p53 tumor-suppressor gene. N Engl J Med 1993; 329:1318-1327.

24 Kawasaki M, Noguchi M, Morikawa A, et al. Nuclear p53 accumulation by small-sized adenocarcinomas of the lung. Pathol Int 1996;46:486-490.

$25 \mathrm{Li} \mathrm{ZH,} \mathrm{Zheng} \mathrm{J,} \mathrm{Weiss} \mathrm{LM,} \mathrm{et} \mathrm{al.} \mathrm{C-k-ras} \mathrm{and} \mathrm{p53}$ mutations occur very early in adenocarcinoma of the lung. Am J Pathol 1994;144:303-309.

26 Westra WH, Offerhaus GJ, Goodman SN, et al. Overexpression of p53 tumor suppressor gene product in primary lung adenocarcinomas is associated with cigarette smoking. Am J Surg Pathol 1993;17:213-220.

27 Hoffmann H. Resected non-small cell lung cancer stage I/II: indication for adjuvant/neoadjuvant therapy? Lung Cancer 2004;45(suppl 2):S91-S97.

28 Pisters KMW, Le Chevalier T. Adjuvant chemotherapy in completely resected non-small cell lung cancer. J Clin Oncol 2005;23:3270-3278.

29 Sakao Y, Sakuragi T, Natsuaki M, et al. Clinicopathological analysis of prognostic factors in clinical IA peripheral adenocarcinoma of the lung. Ann Thorac Surg 2003;75:1113-1117.

30 Susuki K, Nagai K, Yoshida J, et al. Prognostic factors in clinical stage I non-small cell lung cancer. Ann Thorac Surg 1999;67:927-932. 